\section{Transposed chickens}

Whereas agricultural advances have traditionally come from selective breeding programs, a number of recent advances in crop and animal farming have arisen from transgenic engineering approaches. Much of these genetic engineering efforts have been aimed at creating disease resistance as well as improved food quality. Despite their economic potential, poultry have not seen the genetic engineering advances found in other livestock in part due to the relatively low efficiency of achieving stable germline transmission of foreign genes. By using the transposable element mariner, originally isolated from the fly Drosophila mauritiana, Sang and colleagues have been able to insert the foreign genetic element in more than $20 \%$ of the injected embryos resulting in stable transmission in a Mendelian manner (see pp. 1013 and 1050).

\section{From one to many}

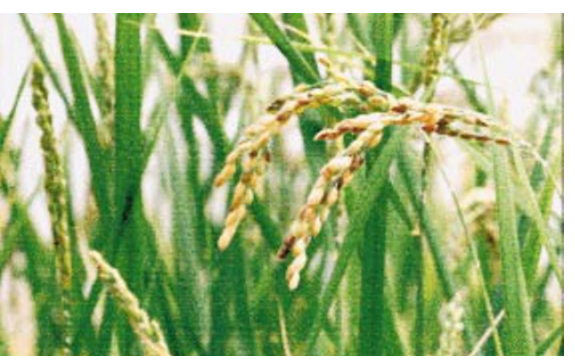

The ability to introduce single genes that confer desirable phenotypes has resulted in the successful commercialization of a variety of transgenic plants. Complex traits and most biosynthetic pathways are governed by a variety of genes working in concert. As an initial step in creating the next generation of metabolically engineered plants, Chen et al. have introduced up to 13 different genetic elements into Japonica rice that in many cases are co-integrated and stable across sexual generations (see pp. 1009 and 1060). The ability to introduce multiple genes in a concerted manner will allow the introduction and modification of entire metabolic pathways rather than merely single genes.

Research briefs written by Philip Bernstein.

\title{
Chimeric brains
}

Stem cells-which are capable of differentiating into a variety of cell types while propagating as multipotential cells-are important tools for the study of development and differentiation. These same characteristics hold promise for gene- or cell replacementtherapies. In particular, human neural stem cells, which could repopulate damaged sites in the central nervous system (CNS), could be used in the treatment of CNS disorders. In two papers in this issue, Flax et al. and

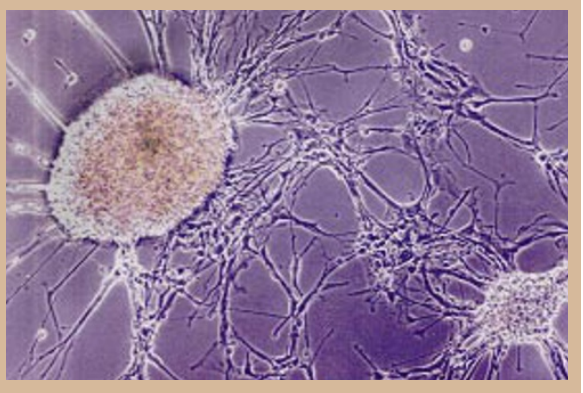
Brüstle et al. (see pp. 1007, 1033, and 1040) have shown the existence of these multipotent cells from the human CNS. Using rodent models they demonstrate the potential of these progenitor cells in repopulating damaged tissue while expressing heterologous genes.

\section{Rational design of an abzyme}

Antibodies are selected to bind with high affinity to a target epitope, and thus enzymes based on antibody structure-abzymes - are attractive in that they should have high specificity for their target. These catatlytically active antibodies are usually generated by selecting antibodies that bind to a stable transition state analogue. Abzymes that cleave nucleic acids have been difficult to generate because of the unavailability of transition state analogues. Fletcher et al, have taken a different approach (see p. 1065). Starting with an existing antibody that recognizes RNA, they converted it to an enzyme by inserting catalytic residues at the binding site. Not yet as robust as other ribonucleases, the creation of an RNA-cleaving abzyme demonstrates an alternative method of creating enzymes with specific activities.

\section{FasL gene therapy}

Despite the early promise of adenovirus based vectors in gene therapy, their widespread therapeutic application has been limited by the propensity of the human immune system to mount an attack against the engineered gene delivery vector. One approach, that has shown only limited success, is to remove the immunogenic proteins of the vector. Zhang et al. have taken a different approach, educating the immune system to ignore the presence of the foreign vector (see pp. 1011 and 1045). By introducing FasL-a ligand that upon interaction with the receptor Fas induces apoptosis-and adenovirus into antigen presenting cells, these cells induce T cell tolerance rather than activation. By tolerizing selected $\mathrm{T}$ cells in a rodent model, immune privilege has been created, allowing prolonged gene therapy using adenovirus vectors.

\section{Screening serum}

Sera from patients infected with a virus contain tell-tale signs of the infection in the form of antibodies. To use these for screening, peptide ligands have to be identified that will bind to all patients' sera. Using sera from several patients infected with hepatitis $\mathrm{C}$ virus, Bartoli et al. have developed a method for screening phage-displayed peptide libraries that includes a DNA hybridization step. By using this second-tier DNA based screening step, phage displaying peptides that are common to multiple HCV-specific sera-which could be used for viral screening-were easily obtained (see p. 1068).

\section{A plant grows in Chernobyl}

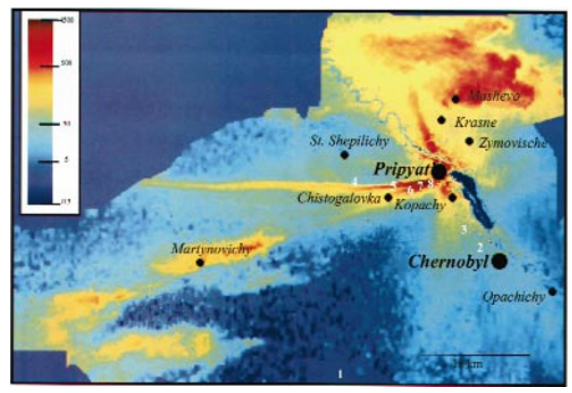

Most notable at the site of the Chernobyl nuclear power plant, radioactive contamination has profound effects on the biosphere. Kovalchuk et al. have engineered Arabidopsis thaliana plants to carry a nonfunctional $\beta$-glucuronidase reporter gene that upon radiation induced recombination turn blue (see pp. 1010 and 1054). These plants can be used to monitor to measure the genetic hazard, to plants and animals, of contaminated sites. 